CUBO A Mathematical Journal Vol.16, Noㅡ, (67-85). October 2014

\title{
On the supersingular loci of quaternionic Siegel space
}

\author{
OLIVER BüLTEL \\ Mathematische Fakultät, \\ Universität Duisburg-Essen, \\ Thea-Leymann Strasse 9, 45127 Essen, Germany. \\ oliver.bueltel@uni-due.de
}

\begin{abstract}
The paper studies the supersingular locus of the characteristic $p$ moduli space of principally polarized abelian 8-folds that are equipped with an action of a maximal order in a quaternion algebra, that is non-split at $\infty$ but split at $p$. The main result is that its irreducible components are Fermat surfaces of degree $p+1$.
\end{abstract}

\section{RESUMEN}

El artículo estudia el lugar supersingular del espacio módulo característico p de abeliano polarizado 8-veces principal que son equipados con una acción de un orden maximal en un álgebra quaterniona, que es no-divisible en $\infty$, pero se divide en $p$. El principal resultados es que los componentes irreducibles son superficies de Fermat de grado $p+1$.

Keywords and Phrases: supersingular abelian varieties, Shimura varieties, orthogonal groups. 2010 AMS Mathematics Subject Classification: 14L05, $14 \mathrm{~K} 10$. 


\section{Introduction}

Let $p$ be a prime number. In 9 . Oort and Li give a description of the supersingular locus $\mathcal{S}_{\mathrm{g}, 1}$ of $\mathcal{A}_{\mathrm{g}, 1} \times \mathbb{F}_{\mathrm{p}}$, the fibre over $\mathrm{p}$ of the Siegel modular variety of principally polarized abelian $\mathrm{g}$-folds. Among their results are that $\mathcal{S}_{g, 1}$ has $\mathrm{H}_{g}(p, 1)$ irreducible components if $g$ is odd and $\mathrm{H}_{g}(1, p)$ if $\mathrm{g}$ is even, and all of these components have dimension $\left[\mathrm{g}^{2} / 4\right]$.

In this paper we study the supersingular locus of certain PEL-moduli spaces $S_{K}$ of type $D_{4}^{\mathbb{H}}$, see body of text for a more precise explanation. These moduli spaces are associated to groups $\mathrm{G}$ that are twists of $\mathrm{GO}(8)$. In the complex analytic context there exist uniformisations by quaternionic Siegel half-spaces, these are tube domains of the shape

$$
\mathfrak{h}=\left\{X+i Y \mid X, Y \in \operatorname{Mat}_{2}(\mathbb{H}), X^{\mathfrak{t}, \mathfrak{l}}=X, Y^{\mathfrak{t}, \mathfrak{l}}=Y>0\right\}
$$

where $\mathbb{H}$ is the non-split quaternion algebra over $\mathbb{R}$, and $\iota$ is the standard involution.

In the algebraic context $S_{K}$ is a 6-dimensional variety parameterizing abelian 8-folds with a particular kind of additional structure, and on a mild assumption on the level structure this variety is smooth. For every prime of good reduction we introduce the usual integral model for this Shimura variety, and we move on to exhibit the geometry of each individual irreducible component of the supersingular locus of the mod p-reduction. Our main result says that these components are Fermat surfaces. This comes as a surprise, because for a more general Shimura variety, the structure of the supersingular locus is usually quite complicated and might not even be smooth, for example this happens in the case of $\mathcal{S}_{3,1}$ the 2-dimensional space of supersingular principally polarized abelian 3-folds, cf. [12, Paragraph(4)], [14, Proposition 2.4], [9, Example(9.4)] or [15] for a very precise exposition. We round off the discussion by turning to the non-supersingular points also, we prove that their p-divisible groups do not have parameters, which is somewhat the exact opposite to their behaviour on the supersingular locus. This too seems unusual, as one sees from the non-supersingular principally polarized abelian 3-folds. These results were already applied in [2] to obtain an Eichler-Shimura congruence relation for $S_{K}$ and for its Shimura divisors.

I am indebted to the referee, and there remains the pleasant task of thanking C.Kaiser, Prof. M.Rapoport, and Prof. T.Wedhorn for remarks on the topic and especially Prof. R.Taylor for much good advice and Prof. F.Oort for some email exchanges.

The paper is organized as follows: Section 2 focuses on local aspects, section 3 on global ones. In subsections 2.1/ 3.1] we sum up definitions and conventions. In subsections 2.2 we explain techniques needed to understand supersingular Dieudonné modules. We apply these techniques to supersingular Dieudonné modules with the particular additional structure under consideration in the subsections 2.3, 2.4 In subsection 2.5 a result on the non-supersingular locus is obtained (Corollary 2.7). 


\section{Structure Theorems on Dieudonné Modules with Pairing}

\subsection{Notions and Notations}

We continue to fix a prime $p$. If $k$ is a perfect field of characteristic $p$, then one denotes by $W(k)$ and $K(k)$ the Witt ring and fraction field thereof. Unless otherwise said, $k$ will be assumed to be algebraically closed. The absolute Frobenius $x \mapsto \chi^{p}$ induces automorphisms $x \mapsto{ }^{F} x$ on $W(k)$ and $K(k)$ which again will be referred to as absolute Frobenii. Recall that a Dieudonné module is a finitely generated, torsion free $W(k)$-module together with a ${ }^{F}$-linear endomorphism $F$ and a $\mathrm{F}^{-1}$-linear endomorphism $\mathrm{V}$ that satisfies $\mathrm{FV}=\mathrm{VF}=\mathrm{p}$. If one tensorizes with $\mathbb{Q}$ one obtains the isocrystal of $M$, this is a finite dimensional $\mathrm{K}(\mathrm{k})$-vector space together with a ${ }^{\mathrm{F}}$-linear bijection $\mathrm{F}$. Dieudonné modules are called isogenous if they give rise to isomorphic isocrystals.

By a pairing on a Dieudonné module $M$ one understands a $W(k)$-bilinear map $\phi: M \times M \rightarrow$ $W(k)$ which satisfies $\phi(x, F y)={ }^{F} \phi(V x, y)$. When thinking of $M$ as the co-variant Dieudonné module of a $p$-divisible group $A$ over $k$, this means that $\phi$ gives rise to a morphism from $A$ to the Serre-dual of $A$. Dieudonné modules with pairings $(M, \phi)$ and $\left(M^{\prime}, \phi^{\prime}\right)$ are called isometric if there exists an isomorphism from $M$ to $M^{\prime}$ taking $\phi$ to $\phi^{\prime}$. The $\operatorname{dimension,~} \operatorname{dim}_{k}(M / V M)$ of a Dieudonné module with non-degenerate pairing is equal to the codimension $\operatorname{dim}_{k}(M / F M)$, the rank of $M$ is necessarily even. A pairing is called antisymmetric if $\phi(x, y)=-\phi(y, x)$ and symmetric if $\phi(x, y)=\phi(y, x)$. In either of these cases we denote $\{x \in M \otimes \mathbb{Q} \mid \phi(M, x) \subset W(k)\}$ by $M^{t}$, if $M=M^{t}$ we say that $\phi$ is perfect.

In [13, Definition(3.1)] the crucial notion of crystalline discriminant of a non-degenerate symmetric pairing is introduced: Say the underlying Dieudonné module has rank $2 n$, and choose a $K(k)$-basis $x_{1}, \ldots, x_{2 n}$ with the additional property $F x_{1} \wedge \cdots \wedge F x_{2 n}=p^{n} x_{1} \wedge \cdots \wedge x_{2 n}$. The determinant $\operatorname{det}\left(\phi\left(x_{i}, x_{j}\right)\right)$, regarded as an element in $K\left(\mathbb{F}_{p}\right)^{\times} /\left(K\left(\mathbb{F}_{p}\right)^{\times}\right)^{2}$, can be checked to be independent of the choice of basis and is called the crystalline discriminant $\operatorname{crisdisc}(M, \phi)$. It only depends on the isogeny class of $M$ allowing one to also write $\operatorname{crisdisc}(M \otimes \mathbb{Q}, \phi)$ for $\operatorname{crisdisc}(M, \phi)$. When fixing once and for all an element $t \in W\left(\mathbb{F}_{p^{2}}\right)^{\times}$with $t^{\sigma}=-t$, the target group $K\left(\mathbb{F}_{p}\right)^{\times} /\left(K\left(\mathbb{F}_{p}\right)^{\times}\right)^{2}$ can be identified with $\left\{1, t^{2}, p, p t^{2}\right\}$ if $p$ is odd and with $\left\{ \pm 1, \pm t^{2}, \pm p, \pm p t^{2}\right\}$ if $p=2$, notice that the kernel of the forgetful map from $K\left(\mathbb{F}_{p}\right)^{\times} /\left(K\left(\mathbb{F}_{p}\right)^{\times}\right)^{2}$ to $K(k)^{\times} /\left(K(k)^{\times}\right)^{2}$ consists of $\left\{1, t^{2}\right\}$. Notice also that the image of $\operatorname{crisdisc}(M, \phi)$ in the group $K(k)^{\times} /\left(K(k)^{\times}\right)^{2}$ is the discriminant of a symmetric pairing in the usual sense of linear algebra, hence is independent of the structure of $M$ as a Dieudonné module. The following characterization of crystalline discriminants within $\left\{1, \mathrm{t}^{2}\right\}$ will be useful, see [13, Corollary(3.5)] for a proof:

Fact 1 (Ogus). Assume that $\phi$ is a non-degenerate symmetric pairing on a Dieudonné module $M$ of rank $2 \mathrm{n}$. Assume also that there exists a $\phi$-isotropic $\mathrm{K}(\mathrm{k})$-subspace $\mathrm{A} \subset \mathrm{M} \otimes \mathbb{Q}$ of dimension n. Then

$$
\operatorname{crisdisc}(M, \phi)=(-1)^{n} t^{2} \operatorname{dim}_{K(k)}(A+F A / A),
$$

in particular, if $\mathrm{A}$ is an isocrystal, then $\operatorname{crisdisc}(M, \phi)=(-1)^{\mathrm{n}}$. 
Recall that the Grassmannian of $n$-dimensional isotropic subspaces of the $\mathrm{K}(\mathrm{k})$-vector space $M \otimes \mathbb{Q}$ has two connected components. Two such spaces $A_{1}$ and $A_{2}$ lie in the same component if and only if the integer $\operatorname{dim}_{k(k)}\left(A_{1}+A_{2} / A_{1}\right)$ is even, we will say that $A_{1}$ and $A_{2}$ have the same parity if this is the case. Thus by the above fact $(-1)^{n} \operatorname{crisdisc}(M, \phi)$ is the trivial element of $\mathrm{K}\left(\mathbb{F}_{\mathrm{p}}\right)^{\times} /\left(\mathrm{K}\left(\mathbb{F}_{\mathrm{p}}\right)^{\times}\right)^{2}$ if and only if the bijection $\mathrm{F}$ does not change the parity of the maximal isotropic subspaces. If $p$ is odd and if $\phi$ is a perfect form on $M$, then one can deduce a further formulation: Pick a maximal isotropic k-subspace $\bar{A} \subset \bar{M}=M / p M$ complementary to $V \bar{M}$. Lift it to $W(k)$ to obtain a maximal isotropic $W(k)$ submodule $A$ of $M$ (the Grassmannian is smooth). Observe that $F \bar{A}=F \bar{M}$ to conclude that $(\bmod 2)$ :

$$
\begin{aligned}
& \operatorname{dim}_{k}(\bar{A}+F \bar{A} / \bar{A}) \\
\equiv & \operatorname{dim}_{k}(\bar{A}+V \bar{M} / \bar{A})+\operatorname{dim}_{k}(V \bar{M}+F \bar{M} / V \bar{M}) \\
\equiv & \operatorname{dim}_{k}(\bar{M} / V \bar{M}+F \bar{M}) \quad(\bmod 2) .
\end{aligned}
$$

The integer $\operatorname{dim}_{k}(M / V M+F M)$ is called the Oort invariant of $M$ and denoted $a(M)$. Thus, we have derived the consequence, implicitely stated in [11, Section 5.3]:

Fact 2 (Moonen). Assume that $\phi$ is a perfect symmetric pairing on the Dieudonné module $\mathrm{M}$ of rank $2 \mathrm{n}$. If $\mathrm{p}$ is odd, then $\operatorname{crisdisc}(\mathrm{M}, \phi)=(-1)^{\mathrm{n}} \mathrm{t}^{2 \mathrm{a}(\mathrm{M})}$.

The Dieudonné module $M$ is called superspecial if it satisfies $F M=V M$, i.e. if $\operatorname{rank}_{W(k)}(M)=$ $2 a(M)$. Superspecial Dieudonné modules may conveniently be described in terms of their skeletons, these are the $W\left(\mathbb{F}_{p^{2}}\right)$-submodules defined by $\tilde{M}=\{x \in M \mid F x=V x\}$. We write $\mathcal{O}_{\mathbb{B}}$ for the ring extension of $W\left(\mathbb{F}_{p^{2}}\right)$, obtained by adjoining an indeterminate $\sigma$ subject to the relations $\sigma^{2}=p$ and $\sigma a={ }^{\mathrm{F}} \mathrm{a} \sigma$, it operates in a self-explanatory way on $\tilde{M}$. As remarked in 8 the assignment $M \mapsto \tilde{M}$ sets up an equivalence of the category of superspecial Dieudonné modules with the cat-

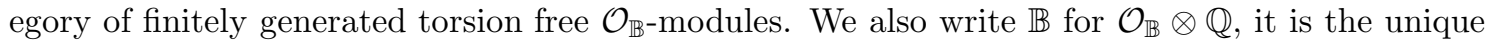
non-split quaternion algebra over $K\left(\mathbb{F}_{\mathfrak{p}}\right)$. Observe that $\mathcal{O}_{\mathbb{B}}$ is the maximal order of $\mathbb{B}$. We let $\mathfrak{m}_{\mathbb{B}}$ be the maximal ideal of $\mathcal{O}_{\mathbb{B}}$, one has $\mathcal{O}_{\mathbb{B}} / \mathfrak{m}_{\mathbb{B}} \cong \mathbb{F}_{p^{2}}$.

We need to put pairings into the picture as follows. If $\phi$ is a pairing on a superspecial Dieudonné module $M$, then one considers a $\mathcal{O}_{\mathbb{B}}$-valued pairing on $\tilde{M}$ defined by:

$$
\Phi(x, y)=\phi(x, \sigma t y)-\phi(x, y) \sigma t
$$

This is $\mathcal{O}_{\mathbb{B}}$-sesquilinear, i.e. satisfies $\Phi(u x, v y)=u \Phi(x, y) v^{\iota}$, for $u, v \in \mathcal{O}_{\mathbb{B}}$. The involution $\iota$ is the standard one, mapping $a+b \sigma$ to ${ }^{F} a-b \sigma$. Conversely any $\mathcal{O}_{\mathbb{B}}$-sesquilinear form arises from a pairing on $M$ in the way described, $\phi$ is non-degenerate/perfect if and only if $\Phi$ is.

Unless otherwise said we assume from now on that $\phi$ is symmetric, in terms of $\Phi$ this means 
$\Phi(y, x)^{\mathfrak{l}}=-\Phi(x, y)$ for all $x, y \in \tilde{M}$. The $\mathcal{O}_{\mathbb{B}}$-module $\tilde{M}$ with form $\Phi$ is called hyperbolic if on a suitable $\mathcal{O}_{\mathbb{B}}$-basis $e_{1}, \ldots, e_{n / 2}, f_{1}, \ldots, f_{n / 2}$ of $\tilde{M}$ one has

$$
\Phi\left(e_{i}, e_{j}\right)=\Phi\left(f_{i}, f_{j}\right)=0 \quad, \quad \Phi\left(e_{i}, f_{j}\right)=w \delta_{i, j}
$$

for some non-zero $w \in \mathcal{O}_{\mathbb{B}}$, uniquely determined only up to multiplication by $\mathcal{O}_{\mathbb{B}}^{\times}$. It turns out that $w=-\sigma^{r}$ t is a very convenient choice as the values of the corresponding form $\phi$ will then read:

$$
\phi\left(e_{i}, f_{j}\right)=\left\{\begin{array}{lll}
0 & r \equiv 0 & (\bmod 2) \\
p^{r-1 / 2} \delta_{i, j} & r \equiv 1 & (\bmod 2)
\end{array}\right.
$$

and

$$
\phi\left(e_{i}, F f_{j}\right)=\left\{\begin{array}{lll}
p^{r / 2} \delta_{i, j} & r \equiv 0 & (\bmod 2) \\
0 & r \equiv 1 & (\bmod 2)
\end{array}\right.
$$

and $\phi\left(e_{i}, e_{j}\right)=\phi\left(f_{i}, f_{j}\right)=0$. Equivalently, $(M, \phi)$ is hyperbolic if and only if the Dieudonné module $M$ allows a decomposition into a direct sum of Dieudonné modules $A$ and $B$ with $\phi(A, A)=$ $\phi(B, B)=0$, and $M^{t}=F^{-r} M$, so that $\phi$ identifies the dual of $A$ with $F^{-r} B$.

\section{$2.2 \quad$ Results of Oort and Li}

A Dieudonné module is called supersingular if it is isogenous to a superspecial one, or equivalently if all its Newton slopes are equal to $1 / 2$. This section is primarily concerned with supersingular Dieudonné modules, so recall some of the techniques which are usually applied to them: If $M$ is supersingular it has a biggest superspecial sub-module $S_{0}(M)$ which one can construct as $S_{0}(M)=\tilde{M} \otimes_{W\left(\mathbb{F}_{p^{2}}\right)} W(k)$. Dually there is $S^{\mathcal{O}}(M)$, the smallest superspecial module containing $M$, see [10, Chapter III.2] for proofs of this.

The following facts on the relation of the lattices $S_{0}(M) \subset M \subset S^{0}(M)$ are basic to the study of supersingular Dieudonné modules. The first of them can be found in [8, Corollary(1.7)], along with more information on the functors $S_{0}$ and $S^{0}$. For the other two facts we refer the reader to [8, Lemma(1.5/1.6)] (or [9, Fact(5.8)]) and [8, 1.10(i)] (or [9, Chapter(12.2)]):

Fact $3(\mathrm{Li})$. Let $\mathrm{M}$ be a supersingular Dieudonné module of rank $2 \mathrm{~g}$ over $\mathrm{W}(\mathrm{k})$. Then one has $\mathrm{F}^{\mathrm{g}-1} \mathrm{~S}^{0}(\mathrm{M})=\sum_{i+j=\mathrm{g}-1} \mathrm{~F}^{i} \mathrm{~V}^{\mathrm{j}} \mathrm{M}$. It follows that $\mathrm{F}^{\mathrm{g}-1} \mathrm{~S}^{0}(\mathrm{M}) \subset \mathrm{S}_{0}(\mathrm{M})$, in particular the length of the $\mathrm{W}(\mathrm{k})$-module $\mathrm{S}^{0}(\mathrm{M}) / \mathrm{S}_{0}(\mathrm{M})$ is bounded by $\mathrm{g}(\mathrm{g}-1)$ and equality is acquired if and only if $a(M)=1$.

Fact $4(\mathrm{Li})$. Let $\mathrm{N}$ be a superspecial Dieudonné module of rank $2 \mathrm{~g}$ over $\mathrm{W}(\mathrm{k})$. Let $\mathrm{x}$ be an element of $\mathrm{N}$. Then one has $\mathrm{S}^{0}(\mathrm{~W}(\mathrm{k})[\mathrm{F}, \mathrm{V}] \mathrm{x})=\mathrm{N}$ if and only if the elements

$$
\mathrm{F}^{\mathrm{g}-1} \mathrm{x}, \mathrm{F}^{\mathrm{g}-2} \mathrm{~V} x, \ldots, \mathrm{FV}^{\mathrm{g}-2} \mathrm{x}, \mathrm{V}^{\mathrm{g}-1} \mathrm{x}
$$

form a basis of the $\mathrm{k}$-vector space $\mathrm{F}^{\mathrm{g}-1} \mathrm{~N} / \mathrm{F}^{\mathrm{g}} \mathrm{N}$. Moreover, an element with this property exists. 
Fact $5(\mathrm{Li})$. Let $\mathrm{M}$ be a supersingular Dieudonné module of rank $2 \mathrm{~g}$ over $\mathrm{W}(\mathrm{k})$. For a non-negative integer $\mathrm{i}$ let

$$
s_{i}=\operatorname{dim}_{k}\left(M \cap F^{i} S^{0}(M) / M \cap F^{i+1} S^{0}(M)\right) .
$$

Then one has $s_{i} \leq s_{i+1}$ and equality holds if and only if $s_{i}=\mathrm{g}$.

The work 9] studies supersingular Dieudonné modules which are equipped with a perfect anti-symmetric form $\psi$. Following their method we notice that we have to incorporate additional structure which by the Morita-equivalence of subsection 3.2 leads to Dieudonné modules $M$ of rank 8 equipped with a symmetric form $\phi$.

Analogous to [9. Proposition(6.1)] we need to analyze the restriction of $\phi$ to $N=S_{0}(M)$, or more generally, a classification of non-degenerate symmetric forms on superspecial Dieudonné modules:

Theorem 2.1. Let $\mathrm{k}$ be an algebraically closed field of characteristic $\mathrm{p} \neq 2$ and let $\mathrm{N}$ be a superspecial Dieudonné module of rank $2 \mathrm{n}$ over $\mathrm{W}(\mathrm{k})$, which is equipped with a non-degenerate symmetric pairing $\phi$. Then $\mathrm{N}$ contains Dieudonné modules $\mathrm{N}_{i}$ of rank 2 , with $\phi\left(\mathrm{N}_{i}, \mathrm{~N}_{\mathfrak{j}}\right)=0$, for $i \neq j$, and $\mathrm{N}=\bigoplus_{i=1}^{\mathrm{n}} \mathrm{N}_{\mathrm{i}}$. Moreover, each $\mathrm{N}_{\mathrm{i}}$ has a $\mathrm{W}(\mathrm{k})$-basis consisting of elements $\mathrm{x}_{i}, \mathrm{Fx}_{\mathrm{i}}=\mathrm{V} \mathrm{x}_{\mathrm{i}}=\mathrm{y}_{\mathrm{i}}$ such that one of the two cases:

(i) $\phi\left(x_{i}, x_{i}\right)=\phi\left(y_{i}, y_{i}\right)=0$, and $\phi\left(x_{i}, y_{i}\right)=p^{n_{i}}$,

(ii) $\phi\left(x_{i}, y_{i}\right)=0, \phi\left(x_{i}, x_{i}\right)=\epsilon_{i} p^{n_{i}}$, and $\phi\left(y_{i}, y_{i}\right)=\epsilon_{i}^{\sigma} p^{n_{i}+1}$,

holds for some integers $n_{i}$ and some elements $\epsilon_{i} \in W\left(\mathbb{F}_{p^{2}}\right)^{\times}$which are unique up to multiplication by elements in $\left(\mathrm{W}\left(\mathbb{F}_{\mathrm{p}^{2}}\right)^{\times}\right)^{2}$. Moreover, the cristalline discriminant can be computed from this decomposition as

$$
\operatorname{crisdisc}\left(N_{i}, \phi\right)=\left\{\begin{array}{ll}
-t^{2} & \left(N_{i}, \phi\right) \text { of type (i) } \\
p t^{2} \epsilon_{i} \epsilon_{i}^{\sigma} & \left(N_{i}, \phi\right) \text { is of type (ii) }
\end{array},\right.
$$

and $\operatorname{crisdisc}(\mathrm{N}, \phi)=\prod_{i=1}^{n} \operatorname{crisdisc}\left(\mathrm{N}_{i}, \phi\right)$.

Proof. The skeleton construction descends $\mathrm{N}$ to a $W\left(\mathbb{F}_{p^{2}}\right)$-Dieudonné module $\tilde{N}$ which at the same time is a $\mathcal{O}_{\mathbb{B}}$-module. As in (2) we consider the $\mathcal{O}_{\mathbb{B}}$-valued sesquilinear form $\Phi$ and diagonalize it as follows: Let $x_{0} \in \tilde{N}$ be an element with $\Phi\left(x_{0}, x_{0}\right)$ of $\mathfrak{m}_{\mathbb{B}}$-adic valuation as small as possible, i.e. such that $\Phi(x, x) \in \mathfrak{m}_{\mathbb{B}}^{r}=\mathcal{O}_{\mathbb{B}} \Phi\left(x_{0}, x_{0}\right)$ for all $x \in \tilde{N}$. By the usual polarization process it follows that $\Phi(x, y)-\Phi(x, y)^{\imath} \in \mathfrak{m}_{\mathbb{B}}^{r}$, and also $\Phi(x, y)+\Phi(x, y)^{\imath} \in \mathfrak{m}_{\mathbb{B}}^{r}$ by replacing $t x$ for $x$. Consequently $\Phi(\tilde{N}, \tilde{N}) \subset \mathfrak{m}_{\mathbb{B}}^{r}$. Therefore we obtain an orthogonal direct sum $\tilde{N}=\left(\tilde{N} \cap\left(\mathbb{B} x_{0}\right)^{\perp}\right) \oplus \mathcal{O}_{\mathbb{B}} x_{0}$, as any $x \in \tilde{N}$ has $\Phi\left(x, x_{0}\right) \Phi\left(x_{0}, x_{0}\right)^{-1}=\alpha \in \mathcal{O}_{\mathbb{B}}$ which allows to write $x$ as a sum of $\alpha x_{0} \in \tilde{N}$ and $x-\alpha x_{0} \in \tilde{N} \cap\left(\mathbb{B} x_{0}\right)^{\perp}$.

Having obtained a decomposition $\tilde{N}=\bigoplus_{i=1}^{n} \tilde{N}_{i}$ we search for basis elements $\tilde{x}_{i} \in \tilde{N}_{i}$ with $\Phi\left(\tilde{x}_{i}, \tilde{x}_{i}\right)$ manageable: In $\tilde{N}_{i} \otimes \mathbb{Q}$ one can certainly find elements $\tilde{x}_{i}$ with $\Phi\left(\tilde{x}_{i}, \tilde{x}_{i}\right) \in W\left(\mathbb{F}_{p^{2}}\right)^{\times} \cup F W\left(\mathbb{F}_{p^{2}}\right)^{\times}$ for example by [16, Chapter 10, Theorem(3.6.(i))]. Observe that the $\mathfrak{m}_{\mathbb{B}}$-adic valuation of $\Phi\left(\tilde{x}_{i}, \tilde{x}_{i}\right)$ must be congruent modulo 2 to $r_{i}=\operatorname{length}_{\mathcal{O}_{\mathbb{B}}} \tilde{N}_{i}^{t} / \tilde{N}_{i}$. Hence after adjusting the $\tilde{x}_{i}$ 's by multiplying 
them by $F^{r_{i} / 2}$, if $r_{i}$ is even, and by $F^{\left(r_{i}-1\right) / 2}$, if $r_{i}$ is odd, one gets generators of the $\mathcal{O}_{\mathbb{B}}$-modules $\tilde{N}_{i}$ on which the sesquilinear form takes values in $F^{r_{i}} W\left(\mathbb{F}_{p^{2}}\right)^{\times}$.

It is clear how to obtain the desired basis $x_{1}, \ldots, x_{n}, y_{1}, \ldots, y_{n}$ from these generators. If $r_{i}$ is even $N_{i}$ will be of type (i) with $n_{i}=r_{i} / 2$, and if $r_{i}$ is odd then $N_{i}$ will be of the type (ii) with $n_{i}=\left(r_{i}-1\right) / 2$.

Remark 2.2. Suppose $\mathrm{N}$ is a superspecial Dieudonné module of rank 2 with a symmetric form $\phi$. Then one checks from the above classification that $(N, \phi)$ is isometric to $(N,-\phi)$. It follows that $N^{\oplus 2}$, the orthogonal direct sum of two copies of $N$, is hyperbolic. One checks this by using the sesquilinear form (2) as $\Phi\left(\left(u_{1}+u_{2}, u_{1}-u_{2}\right),\left(v_{1}+v_{2}, v_{1}-v_{2}\right)\right)=\left(u_{1}+u_{2}\right) w\left(v_{1}+v_{2}\right)^{\iota}-$ $\left(u_{1}-u_{2}\right) w\left(v_{1}-v_{2}\right)^{\iota}=\left(2 u_{1} w\right) v_{2}^{\iota}-u_{2}\left(2 v_{1} w\right)^{\iota}$. (cf. [9, Remark(6.1)] for the analog in the anti-symmetric setting)

For later use we note an immediate corollary:

Corollary 2.3. Let $\left(\mathrm{N}_{1}, \phi_{1}\right)$ and $\left(\mathrm{N}_{2}, \phi_{2}\right)$ be supersingular Dieudonné modules of rank two, equipped with symmetric pairings. There exists an isometry between them if and only if the following holds:

$$
\begin{aligned}
\operatorname{length}_{W(k)} N_{1}^{t} / N_{1} & =\operatorname{length}_{W(k)} N_{2}^{t} / N_{2} \\
\operatorname{crisdisc}\left(N_{1}, \phi_{1}\right) & =\operatorname{crisdisc}\left(N_{2}, \phi_{2}\right) .
\end{aligned}
$$

Consequently for any non negative integer $\mathrm{n}$, there is only one isometry class of rank two supersingular Dieudonné modules with pairing $(\mathrm{N}, \phi)$ where length ${ }_{\mathrm{W}(\mathrm{k})} \mathrm{N}^{\mathrm{t}} / \mathrm{N}=2 \mathrm{n}$. There are two such classes of modules with pairing where length $\mathrm{W}_{(\mathrm{k})} \mathrm{N}^{\mathrm{t}} / \mathrm{N}=2 \mathrm{n}+1$.

\subsection{Classification of symmetric Dieudonné modules}

This section is the core of the work, we give a classification of Dieudonné modules with the additional structure of interest.

Theorem 2.4. Let $\mathrm{M}$ be a supersingular Dieudonné module over $\mathrm{W}(\mathrm{k})$ with perfect symmetric pairing $\phi$. Assume that:

$$
\begin{aligned}
& \operatorname{rank}_{W(k)} M=8 \\
& \operatorname{crisdisc}(M, \phi)=1
\end{aligned}
$$

Consider $\mathrm{S}^{0}(\mathrm{M})=\mathrm{N}$, the smallest superspecial Dieudonné lattice in $\mathrm{M} \otimes \mathbb{Q}$, which contains $\mathrm{M}$. Choose a decomposition $\mathrm{N}=\bigoplus_{i=1}^{4} \mathrm{~N}_{i}$ with properties as granted by Theorem 2.1, and with $\mathrm{S}_{0}(M)=\mathrm{N}^{\mathrm{t}}=\bigoplus_{i=1}^{4} \mathrm{~F}^{\mathrm{r}_{i}} \mathrm{~N}_{\mathrm{i}}$ for integers $\mathrm{r}_{1} \leq \mathrm{r}_{2} \leq \mathrm{r}_{3} \leq \mathrm{r}_{4}$. Then $\left(\mathrm{r}_{1}, \mathrm{r}_{2}, \mathrm{r}_{3}, \mathrm{r}_{4}\right)$ is one of

(i) $(0,0,0,0)$

(ii) $(1,1,1,1)$ 
(iii) $(0,2,2,2)$

(iv) $(2,2,2,2)$,

moreover, there exists a superspecial Dieudonné lattice $\mathrm{Q}$, which contains FM and satisfies

(a) $\mathrm{Q}^{\mathrm{t}}=\mathrm{Q}$

(b) $\operatorname{dim}_{k}(M / M \cap Q)=\operatorname{dim}_{k}(Q / M \cap Q)=1$.

If $\mathrm{M}$ is of the form (iii) or (iv), then the superspecial Dieudonné lattice $\mathrm{Q}$, satisfying (a) and (b) is unique.

Proof. For the proof we need two auxiliary lemmas:

Lemma 2.5. Let the assumptions on $\mathrm{M}$ be as in the above theorem, then there exist two different indices $i_{1}$ and $i_{2}$ such that $\mathrm{N}_{i_{1}}$ and $\mathrm{N}_{i_{2}}$ are isometric.

Proof. If an even integer $r$ occurs twice amongst the various $r_{i}$ 's one is done, and if an odd integer $r$ occurs three times one is done as well, use the pigeon hole principle and Corollary 2.3 . The condition on the discriminant forces the number of indices $i$ with $r_{i}$ odd to be even. This means that one is left with checking the lemma for the $r_{i}$-quadruples $(0,1,2,3),(0,2,3,3),(0,1,1,2)$, and $(1,1,3,3)$.

The three quadruples with $r_{1}=0$ do not arise, because otherwise $M$ would be an orthogonal direct sum of $N_{1}$ and some supersingular Dieudonné module $M^{\prime}$ of rank 6 and equipped with a perfect symmetric form $\phi^{\prime}$. Applying Fact 2 to $M^{\prime}$ would give that $M^{\prime}$ has Oort invariant 1 or 3 , as $\operatorname{crisdisc}\left(M^{\prime}\right)=\operatorname{crisdisc}\left(N_{1}\right)=-t^{2}$. Fact 3 applied to $M^{\prime}$ would further imply that the elementary divisors of $S^{0}\left(M^{\prime}\right) / S_{0}\left(M^{\prime}\right)$ are either all 0 or all equal to 2 . Hence the elementary divisors of $S^{0}(M) / S_{0}(M)$ would be $(0,0,0,0)$ or $(0,2,2,2)$.

It remains to do the $\left(r_{1}, r_{2}, r_{3}, r_{4}\right)=(1,1,3,3)$-case. Assume that no two of the $N_{i}^{\prime}$ s were isometric. This would lead to a basis $x_{i}, F x_{i}=V x_{i}=y_{i}$ with

$$
\begin{aligned}
& \phi\left(x_{1}, x_{1}\right)=p^{-1}, \phi\left(y_{1}, y_{1}\right)=1 \\
& \phi\left(x_{2}, x_{2}\right)=\epsilon p^{-1}, \phi\left(y_{2}, y_{2}\right)=\epsilon^{\sigma} \\
& \phi\left(x_{3}, x_{3}\right)=p^{-2}, \phi\left(y_{3}, y_{3}\right)=p^{-1} \\
& \phi\left(x_{4}, x_{4}\right)=\epsilon p^{-2}, \phi\left(y_{4}, y_{4}\right)=\epsilon^{\sigma} p^{-1}, \\
& \text { other products }=0,
\end{aligned}
$$

and with $\epsilon$ some non-square in $W\left(\mathbb{F}_{p^{2}}\right)^{\times}$. The module $M$ has to contain an element of the form $\alpha_{1} x_{1}+\alpha_{2} x_{2}+\alpha_{3} x_{3}+\alpha_{4} x_{4}+\beta_{3} y_{3}+\beta_{4} y_{4}$ such that $\beta_{i}, \alpha_{i} \in W(k)$ but not both of $\alpha_{3}$ and $\alpha_{4}$ in $\mathrm{pW}(\mathrm{k})$. As

$$
\phi(x, x)=p^{-1}\left(\alpha_{1}^{2}+\epsilon \alpha_{2}^{2}+\beta_{3}^{2}+\epsilon^{\sigma} \beta_{4}^{2}\right)+p^{-2}\left(\alpha_{3}^{2}+\epsilon \alpha_{4}^{2}\right)
$$


one has $\alpha_{3}^{2}+\epsilon \alpha_{4}^{2} \equiv 0(\bmod p)$, but as

$$
\phi\left(x, F^{2} x\right)=\alpha_{1} \alpha_{1}^{\sigma^{2}}+\epsilon \alpha_{2} \alpha_{2}^{\sigma^{2}}+\beta_{3} \beta_{3}^{\sigma^{2}}+\epsilon^{\sigma} \beta_{4} \beta_{4}^{\sigma^{2}}+p^{-1}\left(\alpha_{3} \alpha_{3}^{\sigma^{2}}+\epsilon \alpha_{4} \alpha_{4}^{\sigma^{2}}\right)
$$

one has $\alpha_{3}^{p^{2}+1}+\epsilon \alpha_{4}^{p^{2}+1} \equiv 0(\bmod p)$ as well. As $\epsilon$ is a non-square in $W\left(\mathbb{F}_{p^{2}}\right)^{\times}$, one has $\epsilon^{\frac{p^{2}-1}{2}} \equiv$ $-1(\bmod p)$, so that we derive the contradiction

$$
\alpha_{3}^{p^{2}+1} \equiv\left(-\epsilon \alpha_{4}^{2}\right)^{\frac{p^{2}+1}{2}} \equiv \epsilon \alpha_{4}^{p^{2}+1} \quad(\bmod p)
$$

Lemma 2.6. With the same notation as in the theorem $r_{i} \leq 2$ for all indices $i$.

Proof. Observe that the lemma would be immediate if one of the $r_{i}$ was zero. So we can assume $0<r_{i}$ for all indices $i$. Pick two indices $i \neq j$ with $r_{i}=r_{j}=r$ and $\operatorname{crisdisc}\left(N_{i}\right)=\operatorname{crisdisc}\left(N_{j}\right)$, according to the previous lemma such indices will exist. Say $(i, j)=(1,2)$ after relabeling, and write according to Remark $2.2 \mathrm{~N}_{1} \oplus \mathrm{N}_{2}=\mathrm{A} \oplus \mathrm{B}$, with $\phi(A, A)=\phi(B, B)=0$ and $A \times F^{r} B \rightarrow W(k)$ a perfect pairing. Consider along the lines of [9, Proposition(6.3)] a $W(k)$-module $M^{\prime}$ which is the image of $\left(B \oplus N_{3} \oplus N_{4}\right) \cap M$ under the projection map $B \oplus N_{3} \oplus N_{4} \rightarrow N_{3} \oplus N_{4}$. $M^{\prime}$ inherits a perfect form and is indeed canonically isomorphic to the sub-quotient $\left(B^{\perp} \cap M\right) /(B \cap M)$ of $M$. One has $\operatorname{crisdisc}\left(M^{\prime}\right)=1$ because $M^{\prime}$ is isogenous to $N_{3} \oplus N_{4}$. By Fact 2 it follows that $M^{\prime}$ is superspecial. Furthermore the proof of [9, Proposition(6.3)] shows that $\mathrm{FN}_{3} \oplus \mathrm{FN}_{4} \subset \mathrm{M}^{\prime} \subset \mathrm{N}_{3} \oplus \mathrm{N}_{4}$. For convenience of the reader we reproduce the argument in loc.cit.: Pick an element in $M$ of the form $x=e+f+n_{3}+n_{4}$ with $e \in \tilde{A}, f \in B, n_{3} \in N_{3}, n_{4} \in N_{4}$ and

$$
S^{0}(M)=S^{0}(W(k)[F, V] x)
$$

it exists due to Fact 4. The elements $F^{3} x, F^{2} V x, F V^{2} x, V^{3} x$ will then form a basis of the k-vector space $F^{3} N / F^{4} N$ so that $F^{3} x-F^{2} V x, F^{2} V x-F V^{2} x, F V^{2} x-V^{3} x$ is a basis of $F^{3}\left(B \oplus N_{3} \oplus N_{4}\right) / F^{4}(B \oplus$ $\mathrm{N}_{3} \oplus \mathrm{N}_{4}$ ). It follows that

$$
\mathrm{S}^{\mathrm{O}}(\mathrm{W}(\mathrm{k})[\mathrm{F}, \mathrm{V}](\mathrm{F}-\mathrm{V}) \mathrm{x})=\mathrm{F}\left(\mathrm{B} \oplus \mathrm{N}_{3} \oplus \mathrm{N}_{4}\right),
$$

but $(F-V) x \in M \cap\left(B \oplus N_{3} \oplus N_{4}\right)$ which projects surjectively onto $M^{\prime}$. As $S^{0}$ is a functor in supersingular Dieudonné modules $\mathrm{FN}_{3} \oplus \mathrm{FN}_{4}$ will be contained in $S^{0}\left(M^{\prime}\right)=M^{\prime}$, and consequently

$$
\mathrm{FN}_{3} \oplus \mathrm{FN}_{4} \subset \mathrm{M}^{\prime}=\mathrm{M}^{\mathrm{t}} \subset \mathrm{F}^{-1} \mathrm{~N}_{3}^{\mathrm{t}} \oplus \mathrm{F}^{-1} \mathrm{~N}_{4}^{\mathrm{t}}=\mathrm{F}^{\mathrm{r}_{3}-1} \mathrm{~N}_{3} \oplus \mathrm{F}^{\mathrm{r}_{4}-1} \mathrm{~N}_{4}
$$

i.e. $r_{3}, r_{4} \leq 2$. However, $r_{3} \equiv r_{4}(\bmod 2)$, as $\operatorname{crisdisc}\left(N_{3}\right)=\operatorname{crisdisc}\left(N_{4}\right)$. Therefore $r_{3}=r_{4}$, as $r_{3}, r_{4} \in\{1,2\}$. Now, note that this does indeed imply that $N_{3}$ is isometric to $N_{4}$.

In order to find that $r_{1}, r_{2} \leq 2$ also, we redo the whole argument, with the roles of $N_{1}$ and $N_{2}$ being replaced by $N_{3}$ and $N_{4}$.

\section{Return to proof of theorem}


We move on to investigate the set of possible quadruples $\left(r_{1}, r_{2}, r_{3}, r_{4}\right)$. If one of the numbers in that sequence is 0 , then Fact 2 shows that we must have either $(0,0,0,0)$ or $(0,2,2,2)$. For the remaining cases $(2,2,2,2),(1,1,1,1)$ and $(1,1,2,2)$ are conceivable. We show that $(1,1,2,2)$ can not arise: Assume we had a Dieudonné module $M$ with $\left(r_{1}, r_{2}, r_{3}, r_{4}\right)=(1,1,2,2)$. It would follow that one had crisdisc $\left(\mathrm{N}_{3}\right)=\operatorname{crisdisc}\left(\mathrm{N}_{4}\right)$ by Corollary 2.3, and so would $\operatorname{crisdisc}\left(\mathrm{N}_{1}\right)=$ $\operatorname{crisdisc}\left(\mathrm{N}_{2}\right)$. By applying Remark 2.2 to both $\mathrm{N}_{1} \oplus \mathrm{N}_{2}$ and $\mathrm{N}_{3} \oplus \mathrm{N}_{4}$ one obtains a basis of $\mathrm{N}$ consisting of say $e_{1}, e_{2}, f_{1}, f_{2}, F e_{1}=V e_{1}, F_{2}=V e_{2}, F_{1}=V f_{1}, F_{f_{2}}=V_{f_{2}}$ and with the only non-zero products being given by

$$
\begin{aligned}
& \phi\left(F e_{1}, F_{1}\right)=1 \\
& \phi\left(e_{1}, f_{1}\right)=\phi\left(e_{2}, F_{2}\right)=\phi\left(f_{2}, F e_{2}\right)=p^{-1} .
\end{aligned}
$$

As $F^{-1} N^{t}$ is superspecial one has $M \not \subset F^{-1} N^{t}$, so that $M$ contains an element of the form $x=$ $\alpha_{1} e_{1}+\beta_{1} f_{1}+\alpha_{2} e_{2}+\beta_{2} f_{2}+\alpha_{3} F_{2}+\beta_{3} F_{2}$, with all $\alpha_{1}, \ldots, \beta_{3} \in W(k)$ and at least one of $\alpha_{2}$ and $\beta_{2}$ a unit. From $\mathrm{Fx} \in \alpha_{2}^{\sigma} \mathrm{Fe}_{2}+\beta_{2}^{\sigma} \mathrm{Ff}_{2}+\mathrm{N}^{\mathrm{t}}$ and $\phi(M, M) \subset W(\mathrm{k})$ one infers $\phi(x, F x) \in$ $p^{-1}\left(\alpha_{2}^{\sigma} \beta_{2}+\beta_{2}^{\sigma} \alpha_{2}\right)+W(k)$, which means that $\alpha_{2}^{\sigma} \beta_{2}+\beta_{2}^{\sigma} \alpha_{2} \equiv 0(\bmod p)$. As we may alter the elements $\alpha_{1}, \ldots, \beta_{3}$ by any element in $\mathrm{pW}(\mathrm{k})$ we can actually assume that $\alpha_{2}^{\sigma} \beta_{2}+\beta_{2}^{\sigma} \alpha_{2}=0$, but then the Dieudonné module

$$
W(k) F x+N^{t}=W(k)\left(\alpha_{2}^{\sigma} \mathrm{Fe}_{2}+\beta_{2}^{\sigma} \mathrm{Ff}_{2}\right)+\mathrm{N}^{\mathrm{t}}
$$

is superspecial contradicting $S_{0}(M)=N^{t}$.

Having done the first assertion of the theorem we now focus on the existence of $Q$. If $M$ is of the form (i), then use Remark 2.2 to write $\mathrm{N}_{1} \oplus \mathrm{N}_{2}$ as direct sum of two isotropic Dieudonné modules $A$ and $B$, between which there is the duality that is induced from the pairing on $\mathrm{N}$. Then one finds that $\mathrm{Q}=\mathrm{F}^{-1} \mathrm{~A} \oplus \mathrm{FB} \oplus \mathrm{N}_{3} \oplus \mathrm{N}_{4}$ is a superspecial Dieudonné lattice that does the job. Similarly for the (ii)-case: Write $N=A_{1} \oplus A_{2} \oplus B_{1} \oplus B_{2}$ with isotropic $A_{i}$ and $B_{i}$, this time equipped with a canonical isomorphism $A_{i}^{t} \cong F B_{i}$. The superspecial lattices

$$
\begin{aligned}
& F A_{1} \oplus F A_{2} \oplus B_{1} \oplus B_{2} \\
& A_{1} \oplus F A_{2} \oplus F B_{1} \oplus B_{2}
\end{aligned}
$$

both satisfy $\mathrm{Q}^{\mathrm{t}}=\mathrm{Q}$, and one of them satisfies property (b) as well.

In the (iii)-case property (a) forces to look at $\mathrm{Q}=\mathrm{N}_{1} \oplus \bigoplus_{i=2}^{4} \mathrm{FN}_{i}$, whereas $\mathrm{Q}=\mathrm{FN}$ in the (iv)-case. We have to show that this module does indeed satisfy (b), to this end observe that the numbers $\operatorname{dim}_{k} M / M \cap Q$ and $\operatorname{dim}_{k} M \cap Q / M \cap F Q$ are nonzero and sum up to 4 , it thus suffices to see that the first of them is strictly smaller than the second. In the (iv)-case this is the content of Fact 5. In the (iii)-case apply Fact 5 to the orthogonal complement of $N_{1}$ in $M$, which is a Dieudonné module of rank 6 with perfect symmetric form.

\subsection{Moduli of symmetric Dieudonné modules}

We consider the graded $\mathbb{F}_{p}$-algebra $R:=\mathbb{F}_{p}\left[A_{1}, A_{2}, B_{1}, B_{2}\right] /\left(\sum_{i=1}^{2} A_{i} B_{i}^{p}+B_{i} A_{i}^{p}\right)$, and its associated projective variety $\bar{X}_{1}:=\operatorname{Proj} R$, which is smooth of relative dimension 2 . Let $\bar{Y}_{1}$ denote the affine 
chart determined by $A_{1} \neq 0$, it is the spectrum of $R_{\left(A_{1}\right)} \cong \mathbb{F}_{p}\left[a_{2}, b_{1}, b_{2}\right] /\left(b_{1}+b_{1}^{p}+a_{2} b_{2}^{p}+b_{2} a_{2}^{p}\right)$, where $a_{2}:=\frac{A_{2}}{A_{1}}, b_{1}:=\frac{B_{1}}{A_{1}}$, and $b_{2}:=\frac{B_{2}}{A_{1}}$. Let $\alpha_{2}, \beta_{1}, \beta_{2} \in W\left(R_{\left(A_{1}\right)}\right)$ be lifts of $a_{2}, b_{1}, b_{2}$ with $\beta_{1}+{ }^{\mathrm{F}} \beta_{1}+\alpha_{2}{ }^{\mathrm{F}} \beta_{2}+\beta_{2}{ }^{\mathrm{F}} \alpha_{2}=0$. Let $T_{\left(A_{1}\right)}$ be the $W\left(R_{\left(A_{1}\right)}\right)$-module $\bigoplus_{i=1}^{4} W\left(R_{\left(A_{1}\right)}\right) t_{i}, L_{\left(A_{1}\right)}$ be the $W\left(R_{\left(A_{1}\right)}\right)$-module $\bigoplus_{i=1}^{4} W\left(R_{\left(A_{1}\right)}\right) l_{i}$, and $M_{\left(A_{1}\right)}$ be $L_{\left(A_{1}\right)} \oplus T_{\left(A_{1}\right)}$. Putting:

$$
\begin{aligned}
& F\left(t_{1}\right)=l_{1} \\
& F\left(t_{2}\right)=l_{2}+\left(\beta_{2}-F^{2} \beta_{2}\right) t_{1} \\
& F\left(t_{3}\right)=l_{3}+\left({ }^{2} \alpha_{2}-\alpha_{2}\right) t_{2}+\left(F^{2} \beta_{2}-\beta_{2}\right) t_{4} \\
& F\left(t_{4}\right)=l_{4}+\left(\alpha_{2}-F^{2} \alpha_{2}\right) t_{1} \\
& V^{-1}\left(l_{1}\right)=t_{1} \\
& V^{-1}\left(l_{2}\right)=t_{2} \\
& V^{-1}\left(l_{3}\right)=t_{3} \\
& V^{-1}\left(l_{4}\right)=t_{4}
\end{aligned}
$$

and using the formula $V^{-1}\left({ }^{V} \alpha x\right)=\alpha F(x)$ defines the structure of a display ([17]) on $M_{\left(A_{1}\right)}$, which moreover has the normal decomposition $L_{\left(A_{1}\right)} \oplus T_{\left(A_{1}\right)}$. One checks that a pairing is given on $M_{\left(A_{1}\right)}$ by $\phi\left(l_{i}, l_{j}\right)=\phi\left(t_{i}, t_{j}\right)=0, \phi\left(l_{i}, t_{j}\right)=\delta_{|i-j|, 2}$. Let also $N=L_{N} \oplus T_{N}$ be the display obtained from the formulas $F\left(t_{i}\right)=l_{i}, V^{-1}\left(l_{i}\right)=t_{i}$ and with pairing defined analogously. Putting:

$$
\begin{aligned}
& \epsilon\left(t_{1}\right)=p t_{3} \\
& \epsilon\left(t_{2}\right)=l_{2}-{ }^{F} \beta_{2} l_{3} \\
& \epsilon\left(t_{3}\right)=t_{1}+{ }^{F} \alpha_{2} t_{2}+{ }^{F} \beta_{1} t_{3}+{ }^{F} \beta_{2} t_{4} \\
& \epsilon\left(t_{4}\right)=-{ }^{F} \alpha_{2} l_{3}+l_{4} \\
& \epsilon\left(l_{1}\right)=p l_{3} \\
& \epsilon\left(l_{2}\right)=p t_{2}-p \beta_{2} t_{3} \\
& \epsilon\left(l_{3}\right)=l_{1}+\alpha_{2} l_{2}+\beta_{1} l_{3}+\beta_{2} l_{4} \\
& \epsilon\left(l_{4}\right)=-p \alpha_{2} t_{3}+p t_{4}
\end{aligned}
$$

defines an embedding of displays $\epsilon_{\left(A_{1}\right)}: M_{\left(A_{1}\right)} \hookrightarrow N \times_{\mathbb{F}_{p}} \bar{Y}_{1}$, satisfying $p \phi(x, y)=\phi(\epsilon(x), \epsilon(y))$. Neither $M_{\left(A_{1}\right)}$ nor $\epsilon_{\left(A_{1}\right)}$ depend on the choice of the lifts $\alpha_{2}, \beta_{1}, \beta_{2}$, which can be checked upon passage to the perfection $R_{\left(A_{1}\right)}^{\text {perf }}$ (here notice that $R_{\left(A_{1}\right)} \rightarrow R_{\left(A_{1}\right)}^{\text {perf }}$ is flat, because $R_{\left(A_{1}\right)}$ is regular). Moreover, the natural action of the Kleinian group on $\bar{X}_{1}$ gives rise to analogous subdisplays of the constant display $N$ regarded over each of the translates $\left\{A_{2} \neq 0\right\},\left\{B_{1}, \neq 0\right\}$, and $\left\{B_{2} \neq 0\right\}$, which in turn gives rise to an inclusion

$$
\epsilon: M \hookrightarrow N \times_{\mathbb{F}_{\mathrm{p}}} \overline{\mathrm{X}}_{1},
$$

of sheaves of displays with respect to the Zariski topology of $\bar{X}_{1}$. This is because the closed points can be used to check the cocycle condition. However, notice that there does not exist a global normal decomposition for $M$. 


\subsection{Miscellaneous}

The study of families of Dieudonné modules with our additional structure within a given isogeny class is meaningful not just for the supersingular one. Recall that every isogeny class of Dieudonné modules can be written as a direct sum of certain simple ones. These are parameterized by pairs of coprime non-negative integers $a$ and $b$ and denoted by $G_{a, b}$, see 10 for details. The isogeny class $\mathrm{G}_{\mathrm{a}, \mathrm{b}}$ contains usually more than one Dieudonné module except if $\mathrm{a}$ or $\mathrm{b}$ is equal to 1 , in which case we are allowed to speak of "the" Dieudonné module of type $G_{a, b}$. We have the following result:

Corollary 2.7. Let $\mathrm{M}$ be a non-supersingular Dieudonné module over $\mathrm{W}(\mathrm{k})$ that is equipped with a perfect symmetric pairing $\phi$. Assume that:

$$
\begin{aligned}
& \operatorname{rank}_{W(k)} M=8 \\
& \operatorname{crisdisc}(M, \phi)=1 .
\end{aligned}
$$

Then $M$ is an orthogonal direct sum $\bigoplus_{i} M_{i}$ where for each of the $\left(M_{i}, \phi\right)$ one of the following alternatives hold:

(i.n) $\left(\mathrm{M}_{i}, \phi\right)$ can be written as $\mathrm{A} \oplus \mathrm{B}$ with mutually dual isotropic Dieudonné modules $\mathrm{A}$ and $\mathrm{B}$, which lie in the isogeny classes $\mathrm{G}_{1, n}$ and $\mathrm{G}_{n, 1}$ for some $\mathrm{n} \in\{0,1,2,3\}$.

(ii) $\left(\mathrm{M}_{i}, \phi\right)$ is supersingular of rank 2 and the perfect pairing thereon is the one described by part (i) of theorem 2.1.

(iii) $\left(\mathrm{M}_{i}, \phi\right)$ is supersingular of rank 4 , and the pairing is such that $\mathrm{S}_{0}\left(\mathrm{M}_{i}\right)$ decomposes into the two Dieudonné modules with pairings described by part (ii) of theorem 2.1.

Moreover, the only combinations which occur are:

- $4 \times($ i. 0$)$

- $2 \times($ i. 0$) \oplus($ i. 1$)$

- (i. 0$) \oplus($ ii $) \oplus$ (iii)

- $($ i. 0$) \oplus($ i. 2$)$

- (i.3)

Proof. We consider the canonical decomposition of $M=M_{0} \oplus M^{\prime} \oplus M_{1}$ into the étale-local, locallocal, local-étale parts. The assertion of the corollary has solely something to do with $M^{\prime}$ which is of some even rank equal to $8-2 f$ and has $\operatorname{crisdisc}\left(M^{\prime}\right)=(-1)^{f}$, here $f$ is the p-rank of $M$. As $M^{\prime}$ is also self-dual it can have only one of the following isogeny types: 
(1) $3 \times \mathrm{G}_{1,1}$

(2) $2 \times \mathrm{G}_{1,1}$

(3) $\mathrm{G}_{1,2} \oplus \mathrm{G}_{2,1}$

(4) $\mathrm{G}_{1,3} \oplus \mathrm{G}_{3,1}$

If $M^{\prime}$ has the above isogeny types 3., or 4. we deduce from [7, Paragraph(16), Satz(3)] and $a\left(M^{\prime}\right)=2$ that $M^{\prime}$ is a direct sum of two Dieudonné modules $A$ and $B$, each with Oort invariant equal to one. The assertion on the pairing is then immediate as neither $A$ nor $B$ is selfdual.

If $M^{\prime}$ has isogeny type $2 G_{1,1}$ it must be superspecial. Then use theorem 2.1 in conjunction with remark 2.2 to check that $M^{\prime}$ has the shape $A \oplus B$ with isotropic $A$ and $B$.

In the case in which the isogeny type of $M^{\prime}$ is $3 G_{1,1}$, we have to work a bit harder: First consider a diagonalization of $S^{0}\left(M^{\prime}\right)=N=\bigoplus_{i=1}^{3} N_{i}$ with $S_{0}\left(M^{\prime}\right)=N^{t}=F^{r_{i}} N_{i}$. An analysis as in the proof of lemma 2.5 yields that $\left(r_{1}, r_{2}, r_{3}\right)=(0,1,1)$, therefore the orthogonal direct summand $\left(N_{1}, \phi\right)$ has a complement with perfect form, say $M^{\prime \prime}$, its Oort invariant is 1 . Therefore $\operatorname{crisdisc}\left(M^{\prime \prime}\right)=t^{2}$. As $r_{2}=r_{3}=1$ this implies that $\operatorname{crisdisc}\left(N_{2}, \phi\right)$, and $\operatorname{crisdisc}\left(N_{3}, \phi\right)$, are the two numbers $p$, and $\mathrm{pt}^{2}$, which is what we wanted.

\section{The Shimura variety $S_{K^{p}}$}

\subsection{Further Notation}

Before we proceed we want to introduce the input data for our PEL-moduli problem: Fix once and for all a quaternion algebra $B$ over $\mathbb{Q}$ and write $R$ for the set of places at which $B$ is non-split. Assume that $\infty \in R$, i.e. that $B_{\mathbb{R}}$ is definite. Let $p$ be a prime which is not in $\{2\} \cup R$ and choose

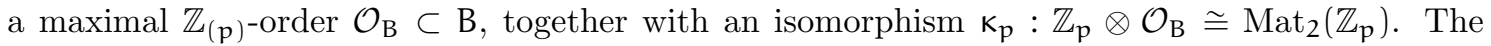
standard involution $\mathrm{b} \mapsto \mathrm{b}^{\mathfrak{l}}=\operatorname{tr}(\mathrm{b})-\mathrm{b}$ preserves $\mathcal{O}_{\mathrm{B}}$ and is positive.

Let $\mathrm{V}$ be a left $\mathrm{B}$-module of rank 4 with non-degenerate alternating pairing satisfying $(\mathrm{b} v, w)=$ $\left(v, b^{\mathfrak{l}} w\right)$. For simplicity we require that the skew-Hermitian B-module $\mathrm{V}$ is hyperbolic in the following sense: We want it to have a B-basis $e_{1}, e_{2}, f_{1}, f_{2}$ such that $\left(\sum_{i=1}^{2} a_{i} e_{i}+b_{i} f_{i}, \sum_{i=1}^{2} a_{i}^{\prime} e_{i}+\right.$ $\left.b_{i}^{\prime} f_{i}\right)=\operatorname{tr}_{B / \mathbb{Q}}\left(\sum_{i=1}^{2} a_{i} b_{i}^{\prime l}-b_{i} a_{i}^{\prime l}\right)$ for all $a_{i}, b_{i}, a_{i}^{\prime}, b_{i}^{\prime} \in B$. Set further $\Lambda_{0}=\bigoplus_{i=1}^{2} \mathcal{O}_{B} e_{i} \oplus \mathcal{O}_{B} f_{i}$, it is a self-dual $\mathcal{O}_{\mathrm{B}}$-invariant $\mathbb{Z}_{(\mathrm{p})}$-lattice in $\mathrm{V}$.

Let $G / \mathbb{Q}$ be the reductive group of all B-linear symplectic similitudes of $V$. This group is a form of $\mathrm{GO}(8)$. Write $K_{p} \subset G\left(\mathbb{Q}_{p}\right)$ for the hyperspecial subgroup consisting of group elements that preserve $\Lambda_{0}$ and let $\mathrm{K}^{\mathrm{p}} \subset \mathrm{G}\left(\mathbb{A}^{\infty, p}\right)$ be an arbitrary compact open subgroup.

Finally we specify a particular $*$-homomorphism $h_{0}: \mathbb{C} \rightarrow \operatorname{End}_{B}\left(V_{\mathbb{R}}\right)$ by the rule $h_{0}(i)\left(\sum_{i=1}^{2} a_{i} e_{i}+\right.$ $\left.b_{i} f_{i}\right)=\sum_{i=1}^{2} b_{i} e_{i}-a_{i} f_{i}$, and $\mathbb{R}$-linear extension. The reflex field of $\left(G, h_{0}\right)$ is equal to $\mathbb{Q}$.

Now, for every connected scheme $S_{\mathrm{Kp}} / \mathbb{Z}_{(\mathrm{p})}$ with a geometric base point $s$ we consider the set of

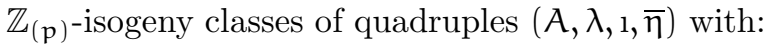


(M1) $A$ is a 8-dimensional abelian scheme over $S$ up to prime-to-p isogeny

(M2) $\lambda: A \rightarrow A^{t}$ is a $\mathbb{Z}_{(p)}^{\times}$-class of prime-to-p polarizations of $A$

$(\mathrm{M} 3) \quad 1: \mathcal{O}_{\mathrm{B}} \rightarrow \operatorname{End}(\mathrm{A}) \otimes \mathbb{Z}_{(\mathfrak{p})}$ is a homomorphism satisfying $1\left(\mathrm{~b}^{\mathfrak{l}}\right)=1(\mathrm{~b})^{*}$, here $*$ is the Rosati involution associated to $\lambda$

$(\mathrm{M} 4) \bar{\eta}$ is a $\pi_{1}(\mathrm{~S}, \mathrm{~s})$-invariant $\mathrm{K}^{\mathrm{p}}$-orbit of $\mathcal{O}_{\mathrm{B}}$-linear isomorphisms $\eta: \mathrm{V} \otimes \mathbb{A}^{\infty, p} \cong \mathrm{H}_{1}\left(\mathrm{~A}_{s}, \mathbb{A}^{\infty, p}\right)$ which are compatible with the alternating form up to scalars.

By geometric invariant theory this functor is representable by a quasi-projective $\mathbb{Z}_{(\mathfrak{p}) \text {-scheme }}$ $S_{K_{p}}$. Moreover, the deformation theory of Grothendieck-Messing shows that $S$ is smooth of relative dimension 6 over $\mathbb{Z}_{(\mathfrak{p})}$, cf. [6, Chapter 5]. See also [6, Chapter 8] for the complex uniformizations of $S_{K p}(\mathbb{C})$.

Finally, let us write $S_{K^{p}}^{s i}\left(\right.$ resp. $\left.S_{K^{p}}^{s p}\right)$ for the subsets $S_{K^{p}} \times \mathbb{F}_{p}^{\text {ac }}$ whose sets of geometric points consist of those quadruples $(A, \lambda, 1, \bar{\eta})$ where $\mathbb{D}\left(A\left[p^{\infty}\right]\right)$ is supersingular (resp. superspecial), here $\mathbb{D}(\mathrm{G})$ denotes the (covariant) Dieudonné module of a $p$-divisible group $G$ over a perfect field. Notice that we always have $\operatorname{crisdisc}(\mathbb{D}(G), \phi)=1$, by $[1$.

\subsection{Morita equivalence}

Let us write $G^{*}$ for the Serre-dual of a $p$-divisible group $G=\bigcup_{l} G\left[p^{l}\right]$ over some base scheme $S$. We will say that $G$ is polarized (resp. anti-polarized) if it is endowed with an isomorphism $\phi$ to its dual which satisfies $\phi=-\phi^{*}$ (resp. $\phi=\phi^{*}$ ). In particular, consider the anti-polarized $p$-divisible groups $G_{1}:=\mathcal{B} \mathcal{T}(M)$ and $G_{0}:=\mathcal{B} \mathcal{T}(N)$, where $M$ and $N$ are as in section 2.4 The emdedding $\epsilon: M \hookrightarrow N \times_{\mathbb{F}_{p}} \bar{X}_{1}$ gives rise to a canonical isogeny $\epsilon: \mathrm{G}_{1} \rightarrow \mathrm{G}_{0} \times_{\mathbb{F}_{\mathrm{p}}} \bar{X}_{1}$ satisfying $\epsilon^{*} \circ \epsilon=p$ id $_{\mathrm{G}_{1}}$

and $\epsilon \circ \epsilon^{*}=p \operatorname{id}_{\mathrm{G}_{0} \times_{\mathbb{F}_{\mathrm{p}}} \bar{X}_{1}}$, notice also that $\operatorname{ker}(\epsilon) \subset \mathrm{G}_{1}[\mathrm{p}]$ and $\operatorname{ker}\left(\epsilon^{*}\right) \subset \mathrm{G}_{0}[\mathrm{p}] \times_{\mathbb{F}_{\mathrm{p}}} \bar{X}_{1}$ are finite, flat, maximal isotropic subgroup schemes of order $p^{4}$.

If an isomorphism $\mathbb{Z}_{\mathbf{p}} \otimes \mathcal{O}_{\mathrm{B}} \stackrel{{ }_{\mathrm{p}}}{\rightarrow} \operatorname{Mat}_{2}\left(\mathbb{Z}_{\mathbf{p}}\right)$ is fixed once and for all, one obtains a Morita-equivalence

$$
(\mathrm{G}, \phi) \mapsto\left(\mathrm{G}^{\oplus 2},\left(\begin{array}{cc}
0 & \phi \\
-\phi & 0
\end{array}\right)\right)
$$

from the category of anti-polarized p-divisible groups to the category of polarized p-divisible groups with Rosati-invariant $\mathcal{O}_{\mathrm{B}}$-action. In this manner one obtains an anti-polarized p-divisible group $(G, \phi)$ from every $S$-valued point on $S_{K^{p}}$, say represented by $(A, \lambda, 1, \bar{\eta})$, by the requirement

$$
\left(A\left[p^{\infty}\right], \psi_{\lambda}\right) \cong\left(G^{\oplus 2},\left(\begin{array}{cc}
0 & \phi \\
-\phi & 0
\end{array}\right)\right),
$$

where $\psi_{\lambda}: A\left[p^{\infty}\right] \rightarrow A\left[p^{\infty}\right]^{*}$ is the $p$-adic Weil-pairing, which is induced from the polarization $\lambda$ : $A \rightarrow A^{t}$. If $S$ is the spectrum of a perfect field of characteric $p$, we always have $\operatorname{crisdisc}(\mathbb{D}(G), \phi)=$ 
1, by [1]. We next want to define a family of morphisms

$$
c_{x, \eta_{p}}: \bar{X}_{1} \times \mathbb{F}_{\mathrm{p}}^{\mathrm{ac}} \rightarrow S_{\mathrm{K}_{\mathrm{p}}} \times \mathbb{F}_{\mathrm{p}}^{\mathrm{ac}}
$$

which are indexed by superspecial $\mathbb{F}_{p}^{a c}$-points $x=(A, \lambda, 1, \bar{\eta})$, equipped with the following additional datum: By a frame for $x$ we mean an isomorphism $\eta_{p}: G_{0} \times_{\mathbb{F}_{p}} \mathbb{F}_{p}^{a c} \rightarrow G$, where $(G, \phi)$ corresponds to $x \in S_{K p}\left(\mathbb{F}_{p}^{a c}\right)$ by the above Morita-equivalence while $\left(G_{0}, \phi_{0}\right)$ is the previously exhibited antipolarized $p$-divisible group. Let us consider the abelian variety which is defined by the exact sequence:

$$
0 \rightarrow \eta_{p}\left(\operatorname{ker}\left(\epsilon^{*}\right)\right)^{\oplus 2} \rightarrow A \stackrel{e^{t}}{\rightarrow} A_{1} \rightarrow 0,
$$

the isotropicity and the $\mathcal{O}_{\mathrm{B}}$-invariance of $\eta_{\mathfrak{p}}\left(\operatorname{ker}\left(\epsilon^{*}\right)\right)^{\oplus 2}$ give rise to a canonical $\mathbb{Z}_{(\mathfrak{p})}^{\times}$-class of prime-to-p polarizations $\lambda_{1}: A_{1} \rightarrow A_{1}^{\mathrm{t}}$, together with a Rosati-invariant operation $1_{1}: \mathcal{O}_{\mathrm{B}} \rightarrow$ $\operatorname{End}\left(A_{1}\right) \otimes \mathbb{Z}_{(\mathfrak{p})}$ and level structure $\bar{\eta}_{1}$, each gotten by transport of structure. Finally one sees that the quadruple $x_{1}=\left(A_{1}, \lambda_{1}, 1_{1}, \bar{\eta}_{1}\right)$ thus obtained constitutes a $\bar{X}_{1} \times \mathbb{F}_{p}^{\text {ac }}$-valued point, whose classifying morphism we define to be (44). It is easy to see that the image of $c_{x, \eta_{p}}$ is a closed subset, whose geometric points consist of exactly those quadruples $\left(A_{1}, \lambda_{1}, 1_{1}, \bar{\eta}_{1}\right)$ which allow an $\mathcal{O}_{B}$-linear isogeny $e: A_{1} \rightarrow A$, wich is compatible with the level structure and satisfies $p \lambda_{1}=e^{t} \circ \lambda \circ e$.

Remark 3.1. Fix $(A, \lambda, 1, \bar{\eta})=x \in S_{K^{p}}^{s p}\left(\mathbb{F}_{p}^{a c}\right)$. Notice, that we have just shown, that the Zariskiclosed subset $c_{x, \eta_{p}}\left(\bar{X}_{1} \times \mathbb{F}_{p}^{a c}\right):=S_{\chi, K^{p}}^{s p}$ does not dependent on the choice of frame.

\subsection{Description of $S_{K^{p}}^{s i}$}

Now, we would like to investigate whether or not $c_{x, \eta_{p}}$ is a closed immersion, the next lemma is a step towards this direction:

Lemma 3.2. Let $\mathrm{x}$ and $\eta_{\mathrm{p}}$ be as above, then $\mathrm{c}_{x, \eta_{\mathrm{p}}}$ induces an injection on the tangentspaces to each geometric point $\mathrm{u} \in \overline{\mathrm{X}}_{1}(\mathrm{k})$, where $\mathrm{k}$ is an arbitrary algebraically closed field of characteristic p.

Proof. Recall that every k-display $\mathrm{P}$ of dimension $\mathrm{d}$ and codimension $\mathrm{c}$ allows structural equations:

$$
\begin{aligned}
& F\left(t_{j}\right)=\sum_{i=1}^{d} u_{i, j} t_{i}+\sum_{i=1}^{c} u_{i+d, j} l_{i} \\
& V^{-1}\left(l_{j}\right)=\sum_{i=1}^{d} u_{i, j+d} t_{i}+\sum_{i=1}^{c} u_{i+d, j+d} l_{i}
\end{aligned}
$$

for some display-matrix

$$
\left(\begin{array}{ccc}
u_{1,1} & \ldots & u_{1, c+d} \\
\vdots & \ddots & \vdots \\
u_{c+d} & \ldots & u_{c+d, c+d}
\end{array}\right)=\mathrm{u} \in \operatorname{GL}(c+d, W(k))
$$


where $t_{1}, \ldots, t_{d}, l_{1}, \ldots, l_{c} \in P$, and $t_{1}+Q, \ldots, t_{d}+Q \in P / Q$ are bases. Let $L$ and $T$ be the $W(k)-$ submodules of $P$ that are generated by $l_{1}, \ldots, l_{c}$ and $t_{1}, \ldots, t_{d}$, and write $J:=\operatorname{Hom}_{W(k)}(L, T)$. Due to the technique of Norman-Oort the isomorphism classes of infinitesimal deformations of $P$ over the ring of dual numbers $k_{D}:=k[s] /\left(s^{2}\right)$ are parameterized by the elements in $J \otimes_{W(k)} k=$ $\operatorname{Hom}_{k}(\mathrm{Q} / \mathrm{pP}, \mathrm{P} / \mathrm{Q})$, in fact each deformation may be described explicitly as follows: Pick a tangent direction $N \in J \otimes W(k) k$, say with $d \times c$-matrix representation

$$
\left(\begin{array}{ccc}
n_{1,1} & \ldots & n_{1, c} \\
\vdots & \ddots & \vdots \\
n_{d, 1} & \ldots & n_{d, c}
\end{array}\right)
$$

(with respect to the two bases above). Write $W\left(s k_{D}\right)$ for the kernel of the natural map from $W\left(k_{D}\right)$ to $W(k)$, and choose elements $\tilde{n}_{i, j} \in W\left(s k_{D}\right)$ whose 0 -th Witt coordinate is equal to the dual number $s n_{i, j}$. Then

$$
\tilde{\mathrm{u}}:=\left(\begin{array}{cccccc}
1 & \ldots & 0 & \tilde{n}_{1,1} & \ldots & \tilde{n}_{1, c} \\
\vdots & \ddots & \vdots & \vdots & \ddots & \vdots \\
0 & \ldots & 1 & \tilde{n}_{\mathrm{d}, 1} & \ldots & \tilde{n}_{\mathrm{d}, \mathrm{c}} \\
0 & \ldots & 0 & 1 & \ldots & 0 \\
\vdots & \ddots & \vdots & \vdots & \ddots & \vdots \\
0 & \ldots & 0 & 0 & \ldots & 1
\end{array}\right) \mathrm{U} \in \operatorname{GL}\left(\mathrm{c}+\mathrm{d}, W\left(k_{\mathrm{D}}\right)\right)
$$

displays an infinitesimal deformation of $\mathrm{P}$, that corresponds to the tangent direction $\mathrm{N}$, in particular it is the trivial deformation if and only of $\mathrm{N}=0$.

Now let $\left(X_{1}: X_{2}: Y_{1}: Y_{2}\right)$ be the homogeneous coordinates of $u \in \bar{X}_{1}(k)$, and fix one of its non-zero tangent directions $u^{\prime} \in \bar{X}_{1}\left(k_{D}\right)$. To finish the proof of the lemma we only have to show that the associated $k_{D}$-display $M_{\mathfrak{u}^{\prime}}$ is a non-trivial infinitesimal deformation (of $M_{\mathfrak{u}}$, i.e. the special fiber of $\left.M_{u^{\prime}}\right)$. Of course we can assume $\left(X_{1}: X_{2}: Y_{1}: Y_{2}\right)=\left(1: x_{2}: y_{1}: y_{2}\right)$ from the start, so let $\left(1: x_{2}+s a: y_{1}-s\left(a y_{2}^{p}-b x_{2}^{p}\right): y_{2}+s b\right)$ be the homogeneous coordinates of $u^{\prime}$, where $(a, b) \in k^{2}-\{(0,0)\}$. Now recall from section 2.4 that the restriction of $M$ to the affine chart Spec $\mathbb{F}_{\mathrm{p}}\left[\mathrm{a}_{2}, \mathrm{~b}_{1}, \mathrm{~b}_{2}\right] /\left(\mathrm{b}_{1}+\mathrm{b}_{1}^{\mathrm{p}}+\mathrm{a}_{2} \mathrm{~b}_{2}^{\mathrm{p}}+\mathrm{b}_{2} \mathrm{a}_{2}^{\mathrm{p}}\right) \subset \overline{\mathrm{X}}_{1}$ has already a normal decomposition and is explicitly displayed in an extremely convenient way, namely by means of the matrix $U=\left(\begin{array}{ll}H & E \\ E & 0\end{array}\right)$, where $\mathrm{E}$ denotes the identity matrix, and where the (so-called 'Hasse-Witt') matrix $\mathrm{H}$ is given by:

$$
\left(\begin{array}{cccc}
0 & \beta_{2}-{ }^{F^{2}} \beta_{2} & 0 & \alpha_{2}-{ }^{F^{2}} \alpha_{2} \\
0 & 0 & F^{2} \alpha_{2}-\alpha_{2} & 0 \\
0 & 0 & 0 & 0 \\
0 & 0 & F^{2} \beta_{2}-\beta_{2} & 0
\end{array}\right),
$$

for certain $\alpha_{2}, \beta_{1}, \beta_{2} \in W\left(\mathbb{F}_{p}\left[a_{2}, b_{1}, b_{2}\right] /\left(b_{1}+b_{1}^{p}+a_{2} b_{2}^{p}+b_{2} a_{2}^{p}\right)\right)$. Now consider the $s k_{D^{-}}$ valued Witt-vectors $\alpha:=\mathfrak{u}^{\prime}\left(\alpha_{2}\right)-\mathfrak{u}\left(\alpha_{2}\right)$ and $\beta:=\mathfrak{u}^{\prime}\left(\beta_{2}\right)-\mathfrak{u}\left(\beta_{2}\right)$, in fact it is easy to see that 
$\mathfrak{u}^{\prime}\left(\beta_{1}\right)-\mathfrak{u}\left(\beta_{1}\right)=-\left(\alpha \mathfrak{u}\left(\beta_{2}\right)^{\sigma}+\beta \mathfrak{u}\left(\alpha_{2}\right)^{\sigma}\right)$, because $\alpha$ and $\beta$ are killed by $F$. Moreover, the 0 th Wittcoordinates of $\alpha$ and $\beta$ are just sa and sb. It follows immediately that $u^{\prime}(U)=\left(\begin{array}{ll}E & \tilde{N} \\ 0 & E\end{array}\right) u(U)$, with $\tilde{\mathrm{N}}$ being the deformation matrix:

$$
\left(\begin{array}{cccc}
0 & \beta & 0 & \alpha \\
0 & 0 & -\alpha & 0 \\
0 & 0 & 0 & 0 \\
0 & 0 & -\beta & 0
\end{array}\right),
$$

whose matrix of Oth Witt-components is clearly nonvanishing.

As a consequence of theorem 2.4 we have:

$$
S_{K^{p}}^{s i}=\bigcup_{x \in S_{K p}^{s p}} S_{x, K^{p}}^{s i}
$$

and $S_{K^{p}}^{s p}$ is a finite set of closed points. It follows from this (or from Grothendieck's specialization theorem [3, p.149]), that $S_{\mathrm{K}^{\mathrm{p}}}^{s i}$ is Zariski closed. Our aim is to describe $S_{\mathrm{K}^{p}}^{s i}$ together with its induced reduced subscheme structure. Let us fix $x \in S_{K^{p}}^{s p}$, which classifies some quadruple $(A, \lambda, 1, \bar{\eta})$, and let $*$ denote the Rosati-involution on the $\mathbb{Q}$-algebra $\operatorname{End}_{B}^{0}(A)$. Let us write $\mathrm{I}_{x} / \mathbb{Q}$ for the group scheme which represents the functor

$$
C \mapsto\left\{g \in\left(\operatorname{End}_{B}^{0}(A) \otimes C\right)^{\times} \mid g g^{t} \in C^{\times}\right\} .
$$

Every full level structure $\eta: V \otimes \mathbb{A}^{\infty, p} \cong H_{1}\left(A, \mathbb{A}^{\infty, p}\right)$ yields an isomorphism

$$
\mathrm{I} \times \mathbb{A}^{\infty, \mathrm{p}} \stackrel{\cong}{\rightrightarrows} \mathrm{G} \times \mathbb{A}^{\infty, p} ; \gamma \mapsto \eta^{-1} \gamma \eta .
$$

Notice that the preimage of $\mathrm{K}^{\mathrm{p}}$ under the above isomorphism depends only on the $\mathrm{K}^{\mathrm{p}}$-orbit of $\eta$, and hence we can define $K_{x}^{p}:=\eta K^{p} \eta^{-1}$ for any $\eta \in \bar{\eta}$, this is again a compact open subgroup of $I_{x}\left(\mathbb{A}^{\infty, p}\right)$. Consider the compact set $\tilde{K}_{p}:=\left\{\gamma \in \mathrm{I}\left(\mathbb{Q}_{\mathfrak{p}}\right) \mid \gamma, \gamma^{-1} \in \mathrm{p}^{-1} \mathbb{Z}_{\mathfrak{p}} \otimes \operatorname{End}_{\mathrm{B}}(\mathrm{A})\right\}$, and let us say that $K^{p}$ is superneat for $x$ if and only if $I_{x}(\mathbb{Q}) \cap \tilde{K}_{p} \times K_{x}^{p}=\{1\}$. The left-hand side is always a finite group, because $\mathrm{I}_{x}$ is anisotropic. In particular $\mathrm{K}^{\mathrm{p}}$ will always contain some a compact open subgroup which is superneat for every $x \in S_{K^{p}}^{s i}$

Lemma 3.3. If $\mathrm{K}^{\mathrm{p}}$ is superneat for $\mathrm{x}$, then (4) is a closed immersion.

Proof. A morphism from a proper $\mathbb{F}_{\mathrm{p}}^{\mathrm{ac}}$-variety to a separated one is a closed immersion if and only if it radicial and injective on the tangent spaces to all $\mathbb{F}_{p}^{\text {ac }}$-valued points, this is elementary and can be proved along the lines of [4, Lemma 7.4.]. In view of lemma 3.2 it suffices to check that (4) is indeed injective on geometric points. Suppose it wasn't. Then there existed $S_{K \mathfrak{k}}(k) \ni x_{1}=\left(A_{1}, \lambda_{1}, 1_{1}, \bar{\eta}_{1}\right)$ which lies in the image of (4) in two different ways. According to the thoughts at the end of subsection 3.2. this means that there existed two degree- $p^{8}$-isogenies $e, e^{\prime}: A_{1} \rightarrow A$ each of which induce the additional structures $\lambda_{1}, 1_{1}, \bar{\eta}_{1}$ from the additional structures $\lambda, 1, \bar{\eta}$ on $A$. It follows immediately that $\operatorname{id}_{A} \neq e^{\prime} \circ e^{-1}$ is in contradiction to $K^{p}$ being superneat for $x$. 
Received: October 2012. Accepted: March 2013.

\section{References}

[1] Bültel, O., 1999, Rational Points on some PEL-stacks, manuscripta math. Volume 99, p.395-410

[2] Bültel, O., 2002, The congruence relation in the non-PEL case, J. reine angew. Math. Volume 544, p.133-159

[3] Grothendieck, A., 1970, Groupes de Barsotti-Tate et cristaux de Dieudonné, Sém. Math. Sup. Volume 45, Presses de l'Univ. de Montreal

[4] Hartshorne, R., 1977, Algebraic Geometry, Graduate Texts in Mathematics Volume 52, Springer Verlag, New York

[5] Katsura, T. and Oort, F., 1987, Families of supersingular abelian surfaces, Compos. Math. Volume 62 , p.107-167

[6] Kottwitz, R., 1992, Points on some Shimura varieties over finite fields, Journal of the AMS Volume 5, p.373-444

[7] Kraft, H., 1975, Kommutative algebraische p-Gruppen, Sonderforschungsbereich Theoretische Mathematik, Universität Bonn

[8] Li, K-Z., 1989, Classification of Supersingular Abelian Varieties, Math. Ann. Band 283, p.333351

[9] Li, K-Z. and Oort, F., 1998, Moduli of Supersingular Abelian Varieties, Lecture Notes in Mathematics Volume 1680

[10] Manin, Y., 1963, The theory of commutative formal groups over fields of positive characteristic, Russ. Math. Surveys Volume 18, p.1-80

[11] Moonen, B., 2001, Group Schemes with Additional Structures and Weyl Group Cosets, in: Moduli of Abelian Varieties, (Eds. Faber, van der Geer, Oort), Progress in Mathematics Volume 195, p. $255-298$

[12] Oda, T. and Oort, F., 1977, Supersingular abelian varieties, Intl. Sympos. on Algebraic Geometry, Kyoto (Ed. Nagata), p.595-621

[13] Ogus, A., 1989, Absolute Hodge Cycles and Crystalline Cohomology, in: Hodge Cycles, Motives, and Shimura varieties (Eds. Deligne, Milne, Ogus, Shih) Lecture Notes in Mathematics Volume 900, p.357-414

[14] Oort, F., 1991, Hyperelliptic supersingular curves, in: Arithmetic Algebraic Geometry, Texel 1989 (Eds. van der Geer, Oort, Steenbrink), Progress in Mathematics Volume 89, p.247-284 
[15] Richartz, M., 1998, Klassifikation von selbstdualen Dieudonnégittern in einem dreidimensionalen polarisierten supersingulärem Isokristall, Bonn thesis

[16] Scharlau, W., 1985, Quadratic and Hermitian Forms, Grundlehren der mathematischen Wissenschaften 270

[17] Zink, T., 2002, The display of a formal p-divisible Group, in: Cohomologies p-adiques et Applications Arithmétiques (I) (Eds. Berthelot, Fontaine, Illusie, Kato, Rapoport) Astérisque Volume 278, p.127-248 